\title{
CÓMO CONSTRUIR UN ESPECTROSCOPIO CASERO CON UN CD
}

\author{
Santiago Heredia Avalos \\ Departament de Física, Enginyeria de Sistemes i Teoria del Senyal, Universitat \\ d'Alacant, Apartat 99, E-03080 Alacant, Spain
}

[Recibido en Abril de 2009, aceptado en Julio de 2009]

Palabras clave: espectroscopía; red de difracción; ciencia recreativa

\section{INTRODUCCIÓN}

Un espectroscopio es un dispositivo capaz de descomponer la luz visible en sus componentes de diferentes colores (longitudes de onda), es decir, en su espectro, mediante un prisma o una red de difracción (Hewitt, 1995). En esta experiencia se construirá un espectroscopio basado en una red de difracción, usando para ello un CD (del inglés, Compact Disk). Con este espectroscopio se puede introducir al estudiante en el análisis espectral y en el origen de los espectros (saltos de electrones entre estados electrónicos), tanto continuos como discontinuos. En Internet pueden encontrarse diversos diseños de espectroscopios caseros (Belmonte, 2001; Chiaverina, 2006; León-Castellá, 2005; Varetti et al., 2009). Sin embargo, el diseño que se presenta en este trabajo aúna algunas ventajas con respecto a estos últimos: no es necesario recortar el CD (evitando el riesgo que supone esto), el diseño es sencillo y fácil de realizar (se facilita una plantilla del mismo), proporciona resultados reproducibles y de una calidad mejorada (pues como se verá más adelante solventa algunos de los problemas que tienen los diseños anteriores) y es bastante robusto.

Un $C D$ es una superficie reflectante que posee una serie de huecos y salientes (en inglés, pits y lands, respectivamente) mediante los cuales se codifica la información. Los pits y lands están distribuidos a lo largo de un surco que describe una espiral desde el radio exterior hacia el radio interior del CD. Si se hiciese un corte radial al $C D$, se observaría que la separación entre dos surcos consecutivos es una cantidad fija, del orden de la longitud de onda de la luz (1.6 $\mu \mathrm{m}$ para los CD y $0.74 \mu \mathrm{m}$ para los DVD). Debido a esta separación, la luz reflejada en dos surcos consecutivos interfiere entre sí dando lugar a máximos en la intensidad luminosa cuya posición en la superficie del $C D$ depende de la longitud de onda de la luz incidente. Así pues, un CD puede utilizarse como una red de difracción por reflexión para construir con él un espectroscopio casero. Aunque en este trabajo el CD sólo se usará como red de difracción por reflexión, merece la pena destacar que también se puede usar el CD como una red de difracción por transmisión (Chiaverina, 2006). 


\section{CONSTRUCCIÓN DEL ESPECTROSCOPIO}

Para realizar esta experiencia se necesita: un $C D$, dos cartulinas tamaño din- $A 4$, el cartón de un rollo de papel higiénico, papel de aluminio, pegamento, cinta aislante negra, cinta Scotch Magic $®$ o similar, tijeras y cúter.

En primer lugar se dibuja o imprime la plantilla mostrada en la figura 1 sobre dos cartulinas tamaño din-A4. Es importante no modificar la escala de esta figura para obtener el tamaño correcto. A continuación se recortan las cartulinas por las líneas continuas usando las tijeras y el cúter para la rendija. Seguidamente se cortan dos tiras de cinta aislante negra y se pegan paralelas a la rendija de $2 \mathrm{~mm}$, de forma que quede una rendija de $\sim 1 \mathrm{~mm}$ de anchura. De esta forma la cinta aislante negra define de forma precisa la anchura de la rendija. Una vez hecho esto, se cortan dos trozos de cinta Scotch Magic $\AA$ y se pegan tapando la rendija por las dos caras de la cartulina. Esta cinta adhesiva mejora significativamente la calidad del espectro, pues hace que la luz que incide sobre la superficie del CD sea difusa, evitando que las líneas espectrales tengan la forma de la fuente luminosa.

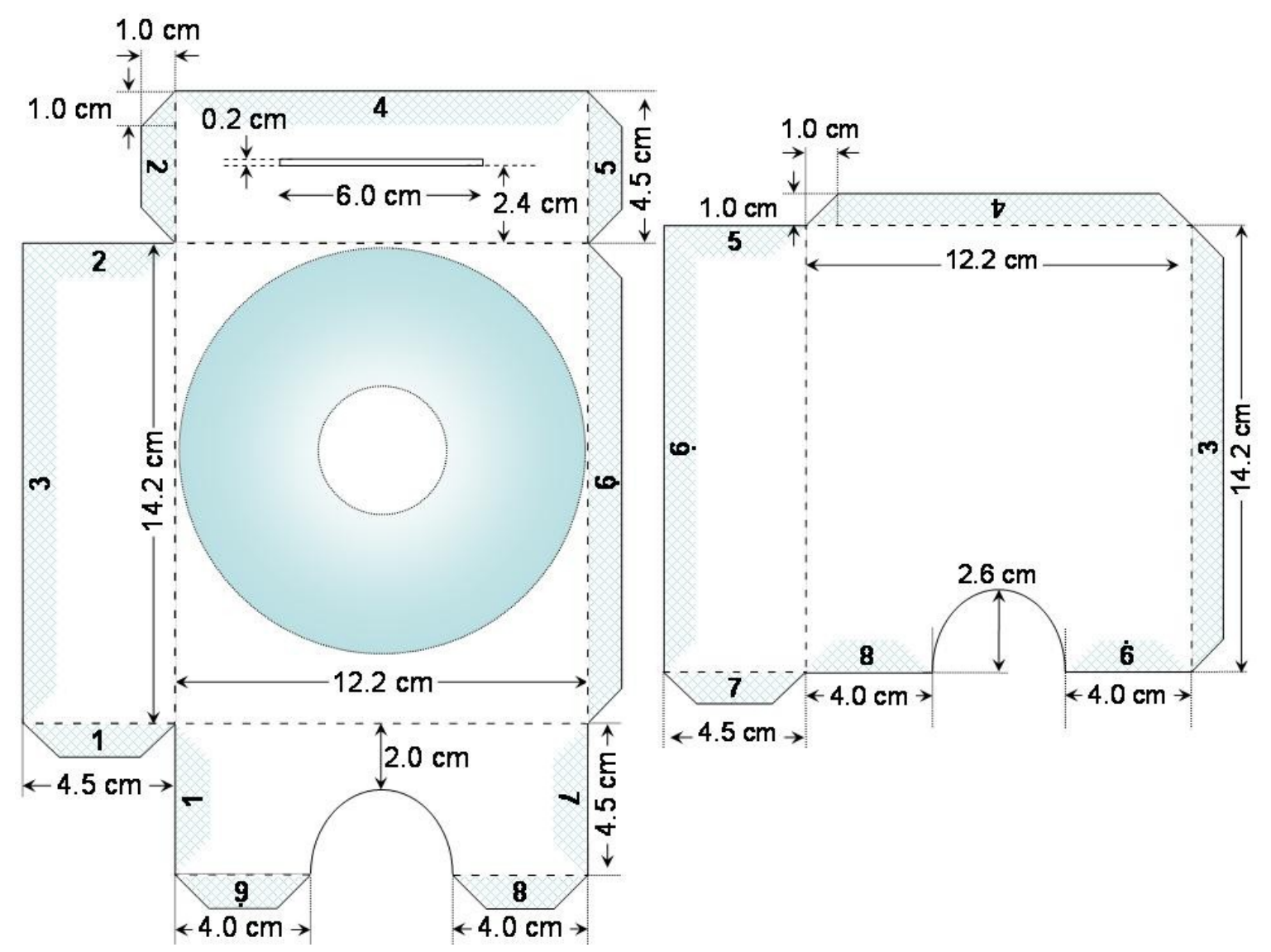

Figura 1.- Plantilla para construir el espectroscopio. Las etiquetas numeradas indican las zonas de las cartulinas que se deben pegar entre sí; el número indica el orden sugerido para que sea más fácil hacerlo. La plantilla está disponible en este enlace. 
A continuación se doblan las cartulinas por las líneas punteadas. Las etiquetas numeradas que se muestran en la figura 1 indican la forma en la que debe pegarse la cartulina; por ejemplo, la pestaña con el número 1 indica que debe pegarse tras la zona sombreada etiquetada con el número 1, y así sucesivamente. El número indica el orden sugerido para pegar la cartulina. Se comienza pegando las pestañas 1 y 2 en los lugares indicados. Seguidamente se pega el CD a la cartulina, de forma que quede al aire la cara

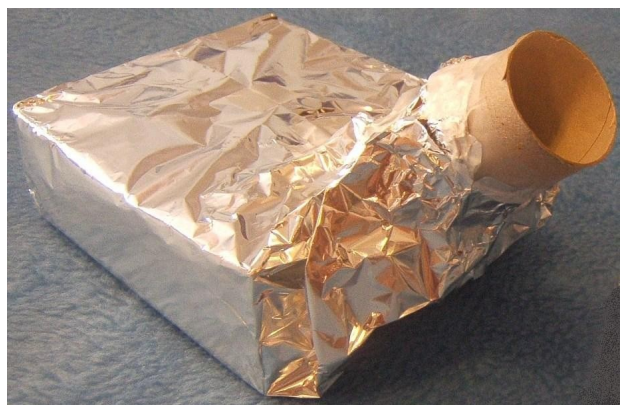

Figura 2. Aspecto del espectroscopio casero. reflectante y que esté colocado lo más cerca posible de la rendija, tal y como se indica en la figura 1 . Se continúa pegando las pestañas con las etiquetas del 3 al 9 . Una vez hecho esto, se introduce el tubo de cartón de un rollo de papel higiénico por el orificio resultante y se pega a la cartulina en la parte superior con un trozo de cinta aislante formando un ángulo de unos $45^{\circ}$, así se fija el tubo a la cartulina y se permite que el ángulo de inclinación sea variable. Finalmente, se envuelve el espectroscopio con papel de aluminio para evitar que penetre la luz por cualquier lugar que no sea la rendija, eliminando de esta forma la contaminación lumínica. En la figura 2 se muestra el aspecto del espectroscopio una vez construido.

El espectroscopio puede acoplarse al objetivo de una cámara fotográfica, si el tamaño del objetivo permite introducirlo en el "ocular" de cartón. En caso contrario habría que construir un manguito casero que actuaría como adaptador.

\section{FUNCIONAMIENTO DEL ESPECTROSCOPIO}

Se puede probar el funcionamiento del espectroscopio orientando la rejilla hacia cualquier fuente luminosa. El espectroscopio descompondrá la luz recibida. En la figura 3 se muestra el aspecto del espectro de varias fuentes luminosas cuando son observadas a través del tubo de cartón, que hace de ocular, del espectroscopio. Las figuras $3 a$ y $3 b$ muestran el espectro obtenido cuando incide sobre la rendija del espectroscopio la luz de una lámpara de bajo consumo ( $\mathrm{CFL}$, del inglés Compact Fluorescent $\operatorname{Lamp}$ ) de color blanco frío y blanco cálido, respectivamente. Se observan varias líneas espectrales bien diferenciadas y un continuo de emisión en la región azul (o roja) del espectro para la lámpara de color blanco frío (o blanco cálido). La figura $3 c$ muestra el espectro de un tubo fluorescente. De nuevo se observan varias líneas espectrales y un continuo de emisión a lo largo de todo el espectro visible. En estos tres casos las líneas espectrales se deben principalmente al vapor de mercurio, mientras que el espectro continuo se debe a las sales de fósforo que recubren el interior del vidrio de este tipo de lámparas. La figura 3d muestra el aspecto del espectro de una bombilla incandescente. En este caso no aparecen líneas espectrales, observándose un espectro continuo asociado a la emisión de radiación de cuerpo negro del filamento incandescente de tungsteno. La figura 3e muestra el espectro de la llama de una vela cuando se añade una pizca de sal común. Se observa un espectro continuo debido a la radiación de cuerpo negro de las partículas incandescentes de 
carbón presentes en la llama, y una línea espectral amarilla que se debe al sodio. Por último, la figura $3 f$ muestra el espectro de la luz solar.[1] A primera vista es un espectro continuo de radiación de cuerpo negro, pero que a diferencia de la bombilla y de la vela posee más color azul en el espectro, al estar a una temperatura mayor. Un análisis más detallado del mismo permite detectar una línea de absorción en la región amarilla del espectro. Esta línea de absorción, que corresponde al sodio presente en el sol, no se observa en la figura $3 f$ por limitaciones técnicas de la cámara fotográfica con la que se realizó la fotografía, aunque sí que puede llegar a apreciarse débilmente a simple vista. Nótese que la posición de la línea de emisión del sodio (figura 3e) debe coincidir con la posición de la línea de absorción del sodio en el espectro solar (figura 3f).

La anchura de las líneas espectrales observadas está definida por la anchura de la rendija $(\sim 1 \mathrm{~mm})$. Así, cuanto menor sea esta anchura, mayor será la capacidad del espectroscopio para observar dos líneas espectrales próximas como separadas, característica denominada capacidad de resolución. Sin embargo, cuanto más estrecha es la rendija, menos luz penetra en el interior del espectroscopio, con la consiguiente disminución en la intensidad de las líneas espectrales observadas. De hecho, el espectro no podrá observarse si la rendija es muy estrecha o si la intensidad luminosa de la fuente es baja. En este último caso, se puede aumentar la intensidad del espectro acercando el espectroscopio a la fuente luminosa.

Estas experiencias permiten explicar que los espectros de emisión y absorción de un átomo son característicos de cada átomo, de forma que es posible identificar la presencia o
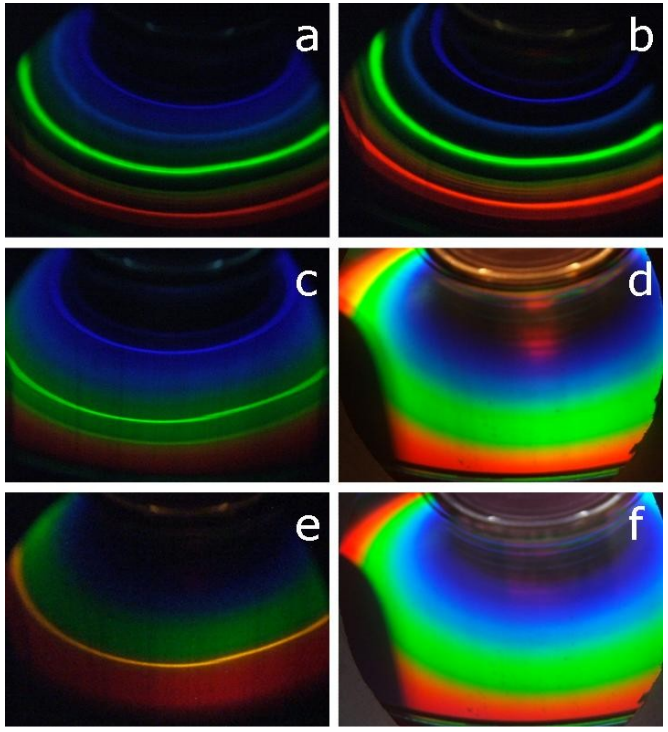

Figura 3. - Espectros obtenidos para diferentes fuentes luminosas: (a) CFL color blanco frío, (b) CFL color blanco cálido, (c) tubo fluorescente, (d) bombilla, (e) llama de vela con sal y (f) Sol. ausencia de un elemento concreto en una fuente luminosa a través de su análisis espectral.

Se puede observar la luz emitida por una lámpara de vapor sodio, como las usadas en el alumbrado público, por un monitor de ordenador de pantalla plana, por un televisor de cañón, por diodos LED (del inglés, Light Emission Diode) de diferentes colores, etc. También se puede probar a añadir a la llama de una vela otras "sustancias", tales como una cerilla o una pizca de bicarbonato sódico. En todos los casos el espectro obtenido es característico de la fuente luminosa en cuestión. Nótese que con este espectroscopio se pueden diferenciar claramente fuentes de luz que a simple vista parecen iguales.

Otra experiencia que puede realizarse con el espectroscopio es observar el espectro de una fuente luminosa después de atravesar un filtro de color. Por ejemplo, se pueden 
usar los separadores de plástico de colores que se usan en los archivadores o cinta aislante de distintos colores a modo de filtros de color. Cuando la luz de una fuente luminosa incide sobre un filtro de color, sólo una parte de la luz es capaz de atravesar el filtro de color, el resto es absorbida por el filtro. Por ejemplo, si se tapa la rendija del espectroscopio con un filtro de color rojo y se observa el espectro de una bombilla incandescente, sólo se percibirá la luz roja, pues la luz que atraviesa el filtro rojo ha perdido las componentes de luz azul y verde. También se puede observar el espectro de absorción de una disolución coloreada. Para ello se observa la luz de una bombilla incandescente que previamente ha pasado a través de la disolución en cuestión. Se pueden usar disoluciones coloreadas de fenolftaleína, extracto de clorofila, extracto de curcumina, etc., todas ellas pueden prepararse fácilmente (Heredia Avalos, 2006). La bombilla incandescente tiene un espectro de emisión continuo. Cuando la luz atraviesa la disolución coloreada, parte de la radiación es absorbida, de forma que se pueden observar las bandas de absorción de la sustancia analizada.

\section{REFERENCIAS BIBLIOGRÁFICAS}

BELMONTE, J. R. (2001). Un espectroscopio sencillo. El rincón de la ciencia, 13. En línea en: http://centros5.pntic.mec.es/ies.victoria.kent/RinconC/Practica/PR-14/PR-14.htm

CHIAVERINA, C. (2006). Spectral analysis: breaking it all down. CoolStuff Newsletter Article, 26. En línea en: http://www.arborsci.com/CoolStuff/cool22.htm

HEREDIA AVALOS, S. (2006). Experiencias sorprendentes de química con indicadores de $\mathrm{pH}$ caseros. Revista Eureka sobre Enseñanza y Divulgación de las Ciencias, 3(1), pp. 89-103. En línea en: http://www.apac-eureka.org/revista/Volumen3/ Numero 3 1/Heredia_2006.pdf

HERNANDEZ, A. (2002). Taller de astronomía. Analiza la luz con tu espectroscopio casero. En línea en: http://eureka.ya.com/astronomia76/ta4.html

HEWITT, P. G. (1995). Física conceptual. Wilmington, Delaware, EEUU: AddisonWesley Iberoamericana.

LEÓN-CASTELLÁ, A., DOHERTY, P., MOYA, L. M. y ROLDÁN, L. (2005). Fundación CIENTEC 2005. Espectroscopio. En línea en: http://www.cientec.or.cr/ciencias/instrumentos/espectroscopio.html

VARETTI, E. L., VARETTI, A. S. y MALLO, L. (2009). Construyendo un espectroscopio. En línea en: http://pagciencia.quimica.unlp.edu.ar/spectrsc.htm

[1] Nunca se debe orientar el espectroscopio directamente al Sol, pues su elevada intensidad luminosa podría dañar la retina del observador. Para observar el espectro del Sol basta con orientar el espectroscopio a cualquier otra parte del cielo.

\section{HOW TO BUILD A HOMEMADE SPECTROSCOPE WITH A CD}

Keywords: spectroscopy; diffraction grating; recreational science 